\title{
Nicotine Enhances Visuospatial Attention by Deactivating Areas of the Resting Brain Default Network
}

\author{
Britta Hahn, ${ }^{1}$ Thomas J. Ross, ${ }^{1}$ Yihong Yang, ${ }^{1}$ Insook Kim, ${ }^{2}$ Marilyn A. Huestis, ${ }^{2}$ and Elliot A. Stein ${ }^{1}$ \\ ${ }^{1}$ Neuroimaging Research Branch and ${ }^{2}$ Chemistry and Drug Metabolism Section, Intramural Research Program, National Institute on Drug Abuse, National \\ Institutes of Health, Baltimore, Maryland 21224
}

\begin{abstract}
Nicotine-induced attentional enhancement is of potential therapeutic value. To investigate the precise attentional function(s) affected and their neuronal mechanisms, the current functional magnetic resonance imaging (fMRI) study used an attention task in which subjects responded to stimuli of high $\left(\mathrm{INT}_{\mathrm{high}}\right.$ ) or low intensity presented randomly in one of four peripheral locations. Central cues of varying precision predicted the target location. In some trials, the cue was not followed by a target, allowing separate analysis of blood oxygenation level-dependent (BOLD) responses to cue. Minimally deprived smokers underwent fast event-related fMRI twice: once with a nicotine patch $(21 \mathrm{mg})$ and once with a placebo patch. Matched nonsmokers were scanned twice without a patch. Behaviorally, nicotine reduced omission errors and reaction time (RT) of valid and invalid cue trials and intra-individual variability of RT and did so preferentially in trials with $\mathrm{INT}_{\text {high }}$. The BOLD signal related to cue-only trials, regardless of cue precision, demonstrated nicotine-induced deactivation in anterior and posterior cingulate, angular gyrus, middle frontal gyrus, and cuneus. These regions overlapped with the so-called "default network," which activates during rest and deactivates with attention-demanding activities. Partial correlations controlling for nicotine plasma levels indicated associations of deactivation by nicotine in posterior cingulate and angular gyrus with performance improvements under $\mathrm{INT}_{\text {high. }}$. Performance and regional activity in the absence of nicotine never differed between smokers and nonsmokers, ruling out a simple reversal of a deprivation-induced state. These findings suggest that nicotine improved attentional performance by downregulating resting brain function in response to task-related cues. Together with the selectivity of effects for $\mathrm{INT}_{\text {high }}$, this suggests a nicotine-induced potentiation of the alerting properties of external stimuli.
\end{abstract}

Key words: nicotine; attention; fMRI; default network; alerting; ASL

\section{Introduction}

Among the beneficial effects of nicotine on performance, reports of its attention-enhancing properties are the most consistent (Stolerman et al., 1995; Newhouse et al., 2004). Because of their potential therapeutic value in chronic disease states characterized by attentional dysfunction (Levin and Rezvani, 2002), the precise attentional functions affected by nicotine and underlying neuronal mechanisms are of growing interest. Nicotine enhanced activity in several brain areas associated with visual attention and arousal during performance of the rapid-visual-informationprocessing (RVIP) task (Lawrence et al., 2002). Because this task combines diverse cognitive components, no link between more specialized neuroanatomy and specific attentional functions could be established. Later studies identified nicotine-induced activity decreases in intraparietal sulcus that accompanied effects

Received Sept. 11, 2006; revised Feb. 21, 2007; accepted Feb. $22,2007$.

This work was supported by the Intramural Research Program of the National Institute on Drug Abuse-National Institutes of Health. We thank Drs. Gordon Shulman and Abraham Snyder for providing the $\left[{ }^{15} 0\right] \mathrm{H}_{2} \mathrm{OPET}$ map of task-induced deactivations.

Correspondence should be addressed to Britta Hahn, Neuroimaging Research Branch, Intramural Research Program, National Institute on Drug Abuse, National Institutes of Health, 5500 Nathan Shock Drive, Baltimore, MD 21224. E-mail: bhahn@intra.nida.nih.gov.

DOl:10.1523/JNEUROSCI.5129-06.2007

Copyright $\odot 2007$ Society for Neuroscience $\quad$ 0270-6474/07/273477-13\$15.00/0 on attentional reorienting (Thiel et al., 2005; Giessing et al., 2006).

Behaviorally, nicotine-induced improvements are most frequently reported in stimulus detection paradigms and vigilance tasks that require continuous maintenance of a simple mental operation or rapid information processing (Koelega, 1993; Bates et al., 1995; Mancuso et al., 2001). Tasks designed to tax the selectivity component of attention such as the Stroop paradigm have been less successful in showing performance enhancement (Parrott and Craig, 1992; Foulds et al., 1996; Poltavski and Petros, 2006). Similarly, animal studies support enhancement of simple stimulus detection (Grilly et al., 2000; Hahn et al., 2002, 2003), but not of discriminatory processes (Turchi et al., 1995; Bushnell et al., 1997), after nicotinic agonist administration. Thus, improved stimulus detection by nicotine does not reflect an enhanced ability to actively direct attention to selected stimulus aspects. In human, monkey, and rat versions of Posner's covert orienting paradigm, nicotine reduced reaction time (RT) costs of invalidly cueing the target location (Witte et al., 1997; Murphy and Klein, 1998; Phillips et al., 2000). Thus, nicotine facilitated stimulus detection at previously unattended locations, possibly reflecting a broadened attentional focus (Bentley et al., 2004) or improved automatic sensory orienting.

This profile of effects may suggest specific facilitation by nicotine of bottom-up processes of attentional resource allocation, 
when attention is spontaneously drawn toward a stimulus driven by its physical onset ("stimulus-driven"). In contrast, top-down attentional control is based on endogenous cognitive processes, is intentional, and is influenced by expectation and current goals (Desimone and Duncan, 1995). Top-down control is engaged when a prominent stimulus feature is ignored in favor of a less prominent one; judging from the negative results with the Stroop paradigm, nicotine may not affect those processes. Because previous studies suggest that distinct neural networks mediate bottom-up and top-down attentional processes (Corbetta and Shulman, 2002), they may be differentially modulated by nicotine.

The aim of the present study was to explore central effects of nicotine that mediate its performance-enhancing effects in a visuospatial attention paradigm. This paradigm differentiates between stimulus detection at attended versus unattended locations and endogenous versus stimulus-driven allocation of attention. Two stimulus intensities were used, postulating that stimulusdriven orienting would be evoked more readily by signals with higher physical salience. Using fast event-related functional magnetic resonance imaging (fMRI), effects of nicotine were explored across and in interaction with specific task conditions.

\section{Materials and Methods \\ Participants}

Seventeen right-handed smokers (11 females) participated in the study. Subjects were 19-49 years of age (mean \pm SD, $33.1 \pm 9.1$ years) and smoked $21 \pm 4.3$ cigarettes/d (range, 17-30) for $15.9 \pm 8.2$ years (range, 4-30). Smokers' intelligence quotient (IQ) as determined by the Wechsler Abbreviated Scale of Intelligence (Wechsler, 1999) was $112.8 \pm 10.5$. An additional 17 right-handed nonsmokers, matched for age (29.1 \pm 7.6 years; range, 19-44), sex (11 females), and IQ (113.8 \pm 10.1$)$, were tested in the absence of drug to compare performance and regional activity between groups at baseline and test for potential withdrawal-related differences in smokers off nicotine. These control subjects reported no nicotine use within the past 12 months. Neuroimaging and performance data from these participants, forming part of a larger group of control subjects, were analyzed in detail in a previous study (Hahn et al., 2006). The reason for studying the effects of nicotine in smokers, apart from ethical concerns, was that nonsmokers initially exposed to nicotine typically display aversive side effects that can overshadow and interfere with the measurement of cognitive effects of nicotine (Heishman et al., 1993; Perkins et al., 1994; Heishman and Henningfield, 2000).

Subjects were recruited from the general population through newspaper advertising, flyers, and referrals and gave written informed consent for a protocol approved by the National Institute on Drug Abuse-Intramural Research Program (NIDA-IRP) Institutional Review Board. Subjects were screened for major medical illnesses, claustrophobia, history of neurological or psychiatric disorders, drug and alcohol abuse, and pregnancy. A urine sample was collected and assessed for common drugs of abuse (Triage; Biosite, San Diego, CA).

\section{Procedure}

The protocol required three separate visits. During the first visit, participants gave informed consent and were trained on two cognitive tasks (one reported elsewhere), initially on a bench computer and then in a mock scanner that mimicked all properties of the MRI scanner. Training in the mock scanner was equal in length to when the tasks were performed in the real scanner. During performance of the current task [the spatial attentional resource allocation task (SARAT)], subjects were repeatedly reminded to keep their eyes focused on the central fixation cross. Participants were also familiarized with the computerized questionnaires to be completed in the scanner and with the wheel response device used for their completion (see below).

Sessions 2 and 3 were identical; however, for smokers, a Nicoderm patch (21 mg/24 h; GlaxoSmithKline, Moon Township, PA) was applied to the upper back in one session, $2-2.5 \mathrm{~h}$ before being loaded into the
MRI scanner, and a placebo patch was applied in the other. The task paradigm reported here began $\sim 3 \mathrm{~h}$ after patch application. The sequence of test sessions was counterbalanced such that nine smokers received placebo in the first and nicotine in the second session, and eight smokers received the opposite sequence. Order of patch application was single-blind. Nonsmokers completed both sessions without any skin patches. The two test sessions were generally scheduled 2-14 d apart, but four of the 34 subjects (three smokers) were tested 29-63 d apart as a result of scheduling availability.

Smokers smoked a cigarette within $1 \mathrm{~h}$ before entering the NIDA-IRP research facilities, with MRI scans starting $\sim 3 \mathrm{~h}$ after their last cigarette. Participants were told not to ingest any alcohol or over-the-counter medication in the $24 \mathrm{~h}$ preceding each session and not to consume more than a half cup of coffee within the preceding $12 \mathrm{~h}$. Before patch application, participants were tested for recent drug use (Triage), for alcohol intake via breath analysis, and for expired breath carbon monoxide (CO) levels (Vitalograph Breath CO monitor; Vitalograph, Lenexa, KS). A blood pressure (BP) and heart-rate (HR) reading was taken before patch application and within $10 \mathrm{~min}$ after the scan. HR was also measured 30, 60, and $120 \mathrm{~min}$ after patch application using a pulse oximeter.

Approximately $5 \mathrm{~min}$ after patch application, subjects underwent retraining of the SARAT on a bench computer $(9 \mathrm{~min})$, first with only validly cued targets to stress the predictive power of the cues, after which invalid and cue-only trials (see below) were reintroduced. Participants then performed a $10 \mathrm{~min}$ refresher training of the final version of the SARAT, during which their eye position on the screen was recorded. Subjects also practiced a finger-tapping procedure in front of the bench computer. After the HR measurement 60 min postpatch, participants were accompanied to a day room, in which they spent the next $1 \mathrm{~h}$ at their leisure. Two hours after patch application, participants were led back to the test facilities and, after the 120 min postpatch HR measurement, entered the MRI facilities. MRI scans started with a brief task of central executive functioning (data not reported). Eight blocks of the SARAT were then performed, separated by 1 min rest periods. This was followed by anatomical scans and a perfusion MRI scan during a 6.5 min fingertapping task described below.

For smoking participants, a venous blood sample $(5 \mathrm{ml})$ was drawn from a forearm vein within $10 \mathrm{~min}$ after each scan. Samples were stored on ice and centrifuged within $2 \mathrm{~h}$ of collection. Plasma was frozen at $-20^{\circ} \mathrm{C}$ until analysis and subsequently assayed for nicotine levels via solid-phase extraction and liquid chromatography-atmospheric pressure chemical-ionization mass spectrometry (Kim and Huestis, 2006).

Subjective state was measured by computerized versions of two selfreport instruments while lying inside the MRI scanner (once just before and once just after the scan session). One instrument assessed subjective feelings in both smokers and control participants by a list of bidirectional visual analog scales shown to be sensitive to mood changes induced by tobacco deprivation (Parrott et al., 1996): tense/relaxed, nervous/calm, energetic/tired, alert/drowsy, contented/irritated, and satisfied/dissatisfied. Additional scales added to cover further nicotine-withdrawal symptoms were as follows: distracted/focused, depressed/happy, and satiated/ hungry. The second instrument was the 12-item version of the Tobacco Craving Questionnaire (TCQ) (Heishman et al., 2003). Smokers rated items such as "A cigarette would taste good right now" on a 7-point scale anchored at "strongly disagree" and "strongly agree." For both scales, participants used a wheel response device to move a cursor on the screen to the desired position on a horizontal bar relative to the two anchors.

\section{The SARAT}

The SARAT has been described and validated previously (Hahn et al., 2006). Participants were required to keep their eyes fixated on a central circle containing a fixation cross (black against a white background of $\left.117 \mathrm{~cd} / \mathrm{m}^{2}\right)$ and to detect a target signal (500 ms) that could occur at any of four peripheral locations marked by empty circles (Fig. 1). With eyes pointed at the center of the fixation cross, based on a viewing distance of $70-80 \mathrm{~cm}$, the center of the target locations was positioned at $10-12.5^{\circ}$ of visual angle. The diameter of the central circle was $2.6-3.0^{\circ}$, and that of the target circles was $1.3-1.5^{\circ}$ of visual angle. Targets consisted of a circle filled with a checkerboard of gray and white squares of $3 \times 3$ pixels each 


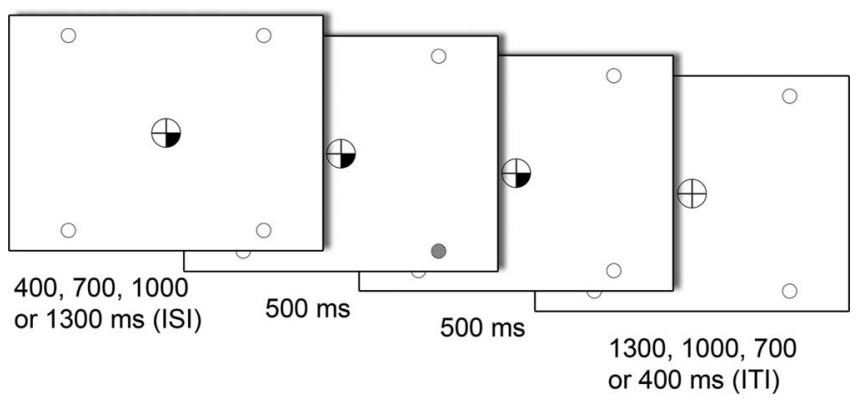

Figure 1. Components of a single target trial in the SARAT. Onset of a central cue preceded target onset by a variable SOA. The target was presented for $500 \mathrm{~ms}$ in the continuing presence of the cue, which remained on display until $500 \mathrm{~ms}$ after target offset. Only screen background was then presented for an intertrial interval (ITI) that varied in length such that total trial duration was always $2700 \mathrm{~ms}$. Cue-only trials differed only in that no target occurred. One, two, three, or all four target locations could be cued at the same time, thus varying the predictability of the target. ISI, Interstimulus interval.

$\left(3\right.$ pixels $=5.2-5.9^{\prime}$ of visual angle; spatial frequency, $\sim 5.4$ cycles $\left./{ }^{\circ}\right)$. Two different target intensities (INT) were tested. For the high target intensity $\left(\mathrm{INT}_{\text {high }}\right)$, gray squares were $80 \%$ gray on a continuum from white to black with a luminance of $10 \mathrm{~cd} / \mathrm{m}^{2}$; for the low target intensity $\left(\mathrm{INT}_{\text {low }}\right)$, they were $20 \%$ gray with a luminance of $73 \mathrm{~cd} / \mathrm{m}^{2}$. Upon target occurrence, subjects were instructed to press a button with their right index finger as quickly as possible.

Cues appeared in the central circle with a variable stimulus-onset asynchrony (SOA) of 400, 700, 1000, or $1300 \mathrm{~ms}$ before target onset and remained on display until $500 \mathrm{~ms}$ after target termination. Trials were averaged across SOAs. The temporal unpredictability of targets required subjects to continuously allocate attention to cued locations in anticipation of a target. Cues consisted of quarters of the fixation circle turning black with their location (12-3, 3-6, 6-9, or 9-12 o'clock) indicating the probable location of the peripheral target. One, two, three, or four quarters could turn black simultaneously; thus, the number of cued locations (CUE) varied the predictability of the target location across trials. Fewer CUES equaled more precise advance information about the target location, whereas increasing CUE increased spatial uncertainty. The cue provided invalid information in $20 \%$ of trials with one, two, or three CUE.

In some trials, the cue was not followed by a target ("cue-only trials"). Valid trials with high-intensity targets, valid trials with low-intensity targets, and cue-only trials were presented with equal frequency. Cueonly trials occurred unpredictably; thus, attention would be allocated to cued locations in anticipation of a target, allowing separate analysis of typical cue-induced blood oxygen level-dependent (BOLD) responses, independent of target-induced processes. In cue-only trials, the cue assumed the same temporal parameters as in target trials, including the variable SOA and continuous display until $500 \mathrm{~ms}$ after the target would have disappeared. To create sufficient temporal jitter for event-related analysis, no-event trials in which no cues or targets were presented were interspersed throughout the task. There were 81 trials in each of the eight runs; 32 valid target trials, 16 cue-only trials, 6 invalid target trials, and 27 no-event trials, resulting in run duration of 3:39 min. All trial types were randomized within runs.

The premise of the task was that cues would instigate endogenous processes in preparation for target detection. Although the general alerting function of the cue toward upcoming targets would not differ with cue precision, more precise advance information about the target location would intensify endogenous processes of spatial attentional selection. Related brain activity is measured in cue-only trials, which are thought to reflect only cue-induced processes. In contrast, bottom-up (stimulus-driven) orienting is engaged when attention is drawn to a location by the physical occurrence of a stimulus. These processes are limited to target trials, with increased unpredictability of the target location augmenting demands on such spontaneous orienting toward the oncoming target. Furthermore, $\mathrm{INT}_{\text {high }}$ targets were expected to evoke stimulus-driven sensory orienting more readily than $\mathrm{INT}_{\text {low }}$ (i.e., the salient physical properties should capture attention more readily).
Controls for nonspecific effects of nicotine on blood flow and neurovascular coupling

In addition to drug-specific effects on neuronal activity, altered BOLD responses after nicotine administration may be attributable to potential nonspecific effects of nicotine on cerebral blood flow or to drug-induced changes in the coupling between neuronal and hemodynamic response dynamics. To test for this possibility, perfusion $\mathrm{fMRI}$ scans were acquired at the completion of the SARAT from six transaxial slices covering both primary motor and visual cortices while subjects performed cyclic $(30 \mathrm{~s}$ on, $30 \mathrm{~s}$ off) bilateral finger tapping. During on periods, a checkerboard of black and white squares that filled the entire screen (spatial frequency, $\sim 0.26 \mathrm{cycles}^{\circ}{ }^{\circ}$ ) and whose contrast reversed three times per second, served as a visual metronome. During off periods, participants fixated on a central cross. The scan started and ended on an off period; thirteen $30 \mathrm{~s}$ periods were presented in total.

\section{Magnetic resonance imaging}

Scanning was performed on a 3 tesla Siemens (Erlangen, Germany) Allegra scanner. Whole-brain functional echoplanar images were acquired for measurement of $\mathrm{T} 2^{*}$-weighted BOLD effects [4 mm sagittal slices; $64 \times 64$ matrix; field of view $(\mathrm{FOV}), 22 \times 22 \mathrm{~cm}$, repetition time (TR), $2.7 \mathrm{~s}$; echo time (TE), $27 \mathrm{~ms}$; flip angle (FA), 85 $5^{\circ}$. In each scanning session, a whole-brain sagittal T1-weighted structural image (magnetization-prepared rapid-acquisition gradient echo) was acquired for anatomical reference $\left(1 \mathrm{~mm}^{3}\right.$ isotropic voxels; TR, $2.5 \mathrm{~s}$; TE, $4.38 \mathrm{~ms}$; $\mathrm{FA}, 8^{\circ}$ ). Perfusion $\mathrm{fMRI}$ scans were acquired in six $7 \mathrm{~mm}$ transaxial slices using a QUIPPS II (Wong et al., 1998) arterial spin labeling (ASL) imaging sequence (FOV, $220 \mathrm{~cm}$; matrix, $64 \times 64$; TR, $3 \mathrm{~s}$; TE, $27 \mathrm{~ms}$; FA, $90^{\circ}$; TI1, $700 \mathrm{~ms}$; TI2, $1400 \mathrm{~ms}$; gap, $10 \mathrm{~mm}$ ). Because of a scanner upgrade, four subjects were scanned with a flow-sensitive alternating inversion recovery-based ASL sequence (Kim, 1995) (TI, 1400; inversion slab thickness, $58 \mathrm{~mm}$ ).

\section{Eye tracking}

Eye position on the screen was recorded using a remote eye-tracking system (IVIEW; Sensomotoric Instruments, Needham, MA) during performance of the $10 \mathrm{~min}$ refresher training that preceded each test session. The purpose was to verify that participants did not refocus their gaze to where they expected the target to come on. The eye tracker consisted of a video camera and infrared light source pointed at the subject's left eye. The percentage of time spent fixating within a central circle of twice the radius of the fixation circle (visual angle, $<3^{\circ}$ ) was calculated. Furthermore, the average distance of subjects' gaze positions from the center of the screen was determined for each cue condition, separately for valid trials and cue-only trials. Absolute distance values were first derived for each cue location and then averaged over locations for each cue condition. Eye tracking was not performed for eight of the 17 control participants because of equipment unavailability. One smoking participant performed only one eye-tracking session on a separate day. For two other smokers, data from one session (the nicotine session in both cases) were corrupted. Analyses comparing the two sessions were thus based on 14 smokers. Eye position was not recorded during scans because of unavailability of an MRI-compatible eye-tracking system.

\section{Data analysis}

Statistical analyses were performed using SPSS 13.0 (SPSS, Chicago, IL).

$B P$ and HR in smokers. BP was analyzed by two-factor repeatedmeasures ANOVA with drug (nicotine and placebo) and time (prepatch and postscan) as within-subject factors. HR underwent the same analysis but included additional levels of the factor time (prepatch, 30, 60, and 120 min postpatch, and postscan).

Subjective self reports. Individual "Parrott" subscales (Parrott et al., 1996) were analyzed by three-factor ANOVA with group (smokers and controls) as a between-subjects factor and session (nicotine vs placebo for smokers; no drug vs no drug for controls) and pre-post (prescan vs postscan) as within-subject factors. TCQ craving scores were obtained only in smokers and were analyzed by two-factor ANOVA (session $\times$ pre-post).

Behavioral performance. Data from the nicotine and placebo scan sessions were analyzed. Trials with RT $<200 \mathrm{~ms}$ or $>1400 \mathrm{~ms}$ were consid- 
ered outliers and excluded from analyses. RT of valid and invalid cue trials (RTval and RTinval) were expressed as averages for each task condition, and omission errors (OMval and OMinval) were expressed as the percentage of target trials in which no response was recorded. The intra-individual variability of RT was calculated as the SD of RTval $\left(\mathrm{RTval}_{\text {stdev }}\right)$. This measure reflects the trial-by-trial consistency of responding; trials were collapsed over CUE to increase the number of trials per condition. In smokers, RTval, RTinval, OMval, and OMinval were analyzed by three-factor ANOVA for repeated measures with drug (nicotine and placebo), CUE (1-4 in valid trials and 1-3 in invalid trials), and INT (high and low) as within-subject factors, followed by two-factor ANOVA and paired $t$ tests where appropriate. RTval ${ }_{\text {stdev }}$ was analyzed by two-factor ANOVA (drug $\times$ INT). To compare performance in the absence of nicotine between smokers and nonsmokers, data from nondrug days were analyzed by three-factor ANOVA with group (smokers and controls) as between- and CUE and INT as within-subject factors. For this analysis, session 1 data were included from nine of the 17 controls, selected randomly, and session 2 data were included from the other eight, thus matching the amount of task preexposure to that in smokers' placebo sessions.

Correlations. For each smoker, RTval, RTinval, and RTval ${ }_{\text {stdev }}$ in the placebo session were subtracted from values in the nicotine session. Similarly, for each brain region that displayed a significant main effect of drug, average activation under placebo was subtracted from that in the nicotine session. The difference values in regional activation and performance underwent partial correlation controlling for nicotine plasma concentrations in both the nicotine and placebo sessions. Plasma concentrations were included as control variables, because they may underlie interindividual variation in both performance and BOLD effects of nicotine, thus potentially creating or enhancing correlations between these variables by acting as a common antecedent cause. Correlations were not established for omission errors, because more than one-third of these values were zero. A significance level of $p<0.005$ was considered significant for correlations.

fMRI. Data were processed using the AFNI software package, version $2.55 \mathrm{j}$ (Cox, 1996). Motion correction was performed by volume registering each three-dimensional volume to a base volume. The time series was then analyzed by voxelwise multiple regression; regressors were expressed as a $\delta$ function convolved with a model hemodynamic response function and its temporal derivative. Trials were always modeled in their entirety. Regressors corresponded to 18 different trial types (1/2/3/4 validly cued locations $\times$ high/low/no target $+1 / 2 / 3$ invalidly cued locations $\times$ high/low target intensity) and to the six motion parameters as nuisance regressors to help account for residual motion. If applicable, one additional nuisance regressor accounted for target trials in which no response was registered and trials with $\mathrm{RT}<200 \mathrm{~ms}$ or $>1400 \mathrm{~ms}$ that were not analyzed. For each subject and each test session, the voxelwise average amplitude of signal change ( $\beta$ value) produced by each trial type was determined relative to baseline. Because of insufficient trial numbers, $\beta$ values for invalid trials were averaged across CUE. The resulting activation maps were resampled to a higher $(1 \mu \mathrm{l})$ resolution, converted to a standard stereotaxic coordinate system (Talairach and Tournoux, 1988), and spatially blurred using a Gaussian $4.2 \mathrm{~mm}$ full-width at halfmaximum isotropic kernel.

In smokers, functional regions of interest (ROIs) were derived by second-level voxelwise two-factor ANOVA for repeated measures across subjects, performed on $\beta$ values for each trial type. ANOVA performed on cue-only trials included drug and cue as within-subject factors. Two separate ANOVAs were performed on valid target trials, one with drug and CUE (collapsed over INT) and one with drug and INT (collapsed over CUE) as factors. ANOVA of invalid target trials included the factors drug and INT. A voxelwise threshold of $p<0.01$ was applied to the $F$ test activation maps, which, combined with a minimum cluster volume size of $321 \mu \mathrm{l}$, yielded an overall false positive $p<0.05$ as determined by Monte Carlo simulation.

To test whether the effects of nicotine may have served to restore a normal functional state aberrant in smokers off nicotine (e.g., because of withdrawal or other adaptations of brain function with chronic nicotine exposure), average levels of activation in nonsmokers were determined within functional ROIs that displayed a main effect of drug in smokers. Baseline levels of activation were compared between groups by independent-samples $t$ tests. Nine smokers received placebo in session 1, and eight received placebo in session 2; accordingly, session 1 data were used from nine and session 2 data were used from eight nonsmoking controls.

To examine the effects of nicotine on BOLD and cerebral blood flow (CBF) responses to visual and motor stimulation, both BOLD- (derived from untagged images) and flow-weighted (derived by voxelwise subtraction of untagged from tagged images) time series were analyzed separately with a box-car regressor after the $30 \mathrm{~s}$ on and off periods convolved with a model hemodynamic response function. ROIs were defined functionally by contrasting on and off periods. Voxelwise BOLDand flow-weighted contrast values were normalized. BOLD contrast values underwent a random-effects one-sample $t$ test against zero. A voxelwise threshold of $p<0.01$ combined with a minimum cluster volume size of $665 \mu$ l yielded an overall false positive $p<0.05$, as determined by Monte Carlo simulation. Eight regions were identified (Table 1), including left and right precentral gyrus, supplementary motor area, and left and right extrastriate cortex. For each session, flow- and BOLD-weighted contrast values were averaged across voxels within each of these five visual and motor areas. For each participant, only voxels that were covered by the six slices in both sessions and that were part of clusters comprising at least 50 voxels were averaged. One participant thus provided no data for the right extrastriate cortex, and three had insufficient coverage of the left extrastriate cortex. Average BOLD and flow-weighted contrast values were compared between the placebo and nicotine sessions by paired $t$ tests across subjects.

To test for effects of nicotine on basal CBF, flow-weighted values during off periods (omitting the first $6 \mathrm{~s}$ ) were converted to approximate quantitative flow (absolute values) by methods described by Wong et al. (1998). The magnetization at equilibrium was estimated from the nonlabeled images based on the saturation recovery relationship of the magnetization. T1 of blood was assumed to be $1300 \mathrm{~ms}$ (Wong et al., 1998). For each participant and each test session, absolute CBF values were averaged across (1) gray and white matter (segmentation of T1-weighted anatomical images was performed using SPM5, http://www.fil.ion. ucl.ac.uk/spm/software) and (2) those functional ROIs that showed robust effects of nicotine during performance of the SARAT. The anterior cingulate cortex (ACC) region was not covered by the six ASL slices in three subjects, and the cuneus was not covered in five subjects. The average values during rest were compared between the nicotine and placebo sessions by paired $t$ tests across subjects.

Head motion during the SARAT was compared between the two test sessions by calculating a composite motion index from the three translational and the three rotational parameters as described by Yang et al. (2005). This index reflects a subject's average head motion between two consecutive TRs. Values did not differ between the nicotine and the placebo sessions $\left[t_{(16)}=1.16\right.$; not significant (NS); paired $t$ test $]$. 


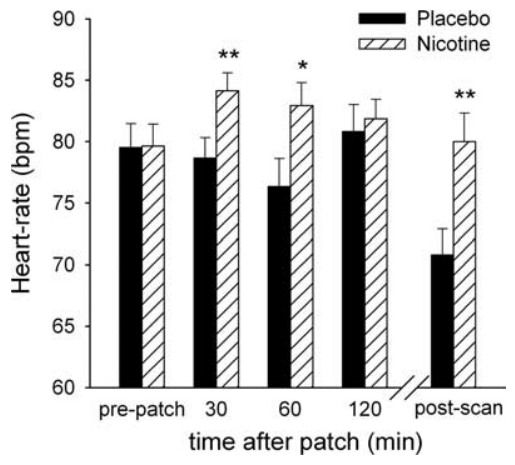

Figure 2. Nicotine increased HR. The effects of nicotine on HR [beats per minute (bpm)] 30 , 60 , and 120 min after nicotine patch application and after completion of the MRI scan are shown. Data are presented as averages \pm SEM over 17 subjects. ${ }^{*} p<0.05$; ${ }^{* *} p<0.01$ (paired $t$ tests).

\section{Results}

\section{Breath CO and plasma nicotine}

On arrival at the research facilities, nonsmokers' breath CO levels were $2.1 \pm 0.6 \mathrm{ppm}$ and $2.8 \pm 2.8 \mathrm{ppm}$ (mean $\pm \mathrm{SD}$ ) in the two visits. Smokers' CO levels were $26.6 \pm 12.9 \mathrm{ppm}$ on placebo test days and $25.5 \pm 8.4 \mathrm{ppm}$ on nicotine test days $\left(t_{(16)}<1\right.$; paired $t$ test). Smokers' plasma nicotine levels were $4.6 \pm 4.0 \mathrm{ng} / \mathrm{ml}$ at completion of the placebo scan and $39.1 \pm 8.9 \mathrm{ng} / \mathrm{ml}$ after the nicotine $\operatorname{scan}\left(t_{(16)}=16.0 ; p<0.001\right)$.

\section{Smokers' vital signs}

Neither systolic nor diastolic BP displayed a main effect of drug or a drug $\times$ time interaction in two-factor ANOVA, indicating that nicotine did not modulate BP. However, HR was significantly elevated by nicotine (Fig. 2), as supported by a drug $X$ time interaction $\left(F_{(4,64)}=5.148 ; p<0.01\right)$. Before patch application, HR did not differ between the nicotine and placebo sessions but was elevated at three of the four postapplication time points. There was no effect of drug 120 min after patch application when baseline HR was elevated, probably because of the recent walk to the test room. The fact that nicotine induced no additional increase under this condition suggests that its effects on HR were baseline dependent.

\section{Eye-tracking data}

During performance of the 10 min refresher training preceding test sessions, the average percentage of all fixations located no farther than $3^{\circ}$ from the center of the screen was $98.6 \pm 2.8 \%$ (mean \pm SD) for smokers $(n=17)$ and $98.0 \pm 3.4 \%$ for controls $(n=9)$. Fixations outside this radius were not systematically oriented toward target locations. Thus, participants were able to maintain central fixation throughout runs. For the 14 smokers whose eye-tracking data were available in both sessions, the percentage of central fixations did not differ between the nicotine and placebo sessions $\left(t_{(13)}=1.34\right.$; NS). The absolute distance of smokers' gaze positions from the screen center did not differ between sessions or cue conditions, either for the vertical or horizontal direction, as confirmed by an absence of any main effects or interactions in two-factor repeated-measures ANOVA.

\section{Subjective state}

Parrott scale

A main effect of group $\left(F_{(1,32)}>26.8 ; p<0.001\right)$ in three-factor ANOVA was obtained in two subscales, reflecting smokers rating themselves as more nervous and tense than control participants. The absence of interactions indicated that this effect did not differ between the placebo and nicotine sessions, suggesting a possible mild anxiogenic effect of patch application and monitoring of vital signs per se rather than a nicotine-withdrawal effect. Significant main effects of pre-post on six subscales $\left(F_{(1,32)}>5.65 ; p<\right.$ $0.05)$ indicated that participants in both groups were more tired and drowsy after than before the scan session, as well as less contented, satisfied, focused, and happy. This appeared to be the general result of performing tasks in the scanner for $\sim 2 \mathrm{~h}$, because there were no interactions with session or with group. A group $\times$ pre-post interaction was seen on the "hungry-satiated" scale $\left(F_{(1,32)}=35.5 ; p<0.001\right)$, with smokers reporting to be significantly less and controls to be more hungry after than before sessions. The only scale to display a session $\times$ group interaction $\left(F_{(1,32)}=5.27 ; p<0.05\right)$ was "focused-distracted," in which a significant effect of session $\left(F_{(1,16)}=5.63 ; p<0.05\right)$ occurred only in smokers, based on reports of being less focused in the placebo than in the nicotine session. Overall, "focused-distracted" ratings of control participants (averaged across sessions) bore greater resemblance to smokers' ratings in the placebo than in the nicotine session, but neither comparison revealed a significant difference $\left({ }_{1} t_{1(16)}^{1}<1\right.$ in both cases).

TCQ-brief

Smokers' craving levels did not differ between the nicotine and placebo sessions $\left(F_{(1,16)}<1\right)$ in two-factor ANOVA. Craving was higher after than before test sessions (main effect of pre-post, $\left.F_{(1,16)}=6.36 ; p<0.05\right)$, but this effect did not differ between the presence or absence of nicotine (pre-post $\times$ session, $F_{(1,16)}<1$ ).

\section{Effects of nicotine on smokers' performance of the SARAT RTval}

RTval (Fig. 3A) increased with increasing CUE and was faster for $\mathrm{INT}_{\text {high }}$ than $\mathrm{INT}_{\text {low }}$, consistent with previous results (Hahn et al., 2006). This was confirmed by main effects of CUE $\left(F_{(3,48)}=\right.$ $14.5 ; p<0.001)$ and INT $\left(F_{(1,16)}=137.5 ; p<0.001\right)$ in twofactor ANOVA. There was no main effect of drug and no significant interaction, but a trend toward a drug $\times$ CUE $\times$ INT interaction $\left(F_{(3,48)}=2.27 ; p=0.093\right)$ prompted additional exploration. Two-factor ANOVA (drug $\times$ CUE) for separate INT revealed no drug effect or interaction. Two-factor ANOVA for separate cue conditions revealed a drug $\times$ INT interaction for trials with one CUE $(1 \mathrm{CUE})\left(F_{(1,16)}=6.44 ; p<0.05\right)$ but not for any other cue condition $\left(F_{(1,16)}<1\right)$. Paired $t$ tests identified a significant nicotine-induced reduction in RTval only in trials with 1 CUE and INT $_{\text {high }}$.

\section{RTinval}

RTinval (Fig. 3B) was overall faster with $\mathrm{INT}_{\text {high }}$ than $\mathrm{INT}_{\text {low }}$ (main effect, $F_{(1,16)}=65.7 ; p<0.001$ ). Three-factor ANOVA also revealed a drug $\times$ INT interaction $\left(F_{(1,16)}=5.59 ; p<0.05\right)$; despite the lower baseline, nicotine reduced RTinval selectively in trials with $\mathrm{INT}_{\text {high }}$. No other main effect or interaction was significant.

OMval

Three-factor ANOVA identified a main effect of INT $\left(F_{(1,16)}=\right.$ 29.8; $p<0.001)$ reflecting lower OMval with $\mathrm{INT}_{\text {high }}$, and a nearsignificant drug $\times$ CUE $\times$ INT interaction $\left(F_{(3,45)}=2.68 ; p<\right.$ 0.058) (Fig. 3C). Separate two-factor ANOVA (drug $\times$ CUE) revealed a main effect of drug for $\operatorname{INT}_{\text {high }}\left(F_{(1,16)}=6.59 ; p<\right.$ $0.05)$ but not $\operatorname{INT}_{\text {low }}\left(F_{(1,16)}<1\right)$. Figure $3 C$ confirms that nicotine reduced OMval across cue conditions only in trials with 
$\mathrm{INT}_{\text {high }}$. The ANOVA further identified a drug $\times$ CUE interaction for INT $_{\text {low }}$ $\left(F_{(3,48)}=2.84 ; p<0.05\right)$, in which OMval increased with CUE under placebo (effect of CUE in one-factor ANOVA, $\left.F_{(3,48)}=5.21 ; p<0.01\right)$ but not under nicotine $\left(F_{(3,48)}<1\right)$.

\section{OMinval}

Apart from a main effect of INT $\left(F_{(1,16)}=\right.$ 28.4; $p<0.001)$ reflecting lower omissions with $\mathrm{INT}_{\text {high }}$, three-factor ANOVA did not reveal any significant main effects or interactions (data not shown).

RTval ${ }_{\text {stdev }}$

$\mathrm{RTval}_{\text {stdev }}$ (Fig. 3D) displayed a main effect of drug $\left(F_{(1,16)}=5.0 ; p<0.05\right)$ in two-factor ANOVA, reflecting reduced $\mathrm{RTval}_{\text {stdev }}$ and thus a more consistent attentional focus with nicotine. A main effect of INT $\left(F_{(1,16)}=51.0 ; p<0.001\right)$ reflected lower RTval stdev $_{\text {with }} \mathrm{INT}_{\text {high }}$. Figure $3 D$ shows that the effect of nicotine was significant only in trials with $\mathrm{INT}_{\text {high }}$, suggesting preferential action under this condition; however, the drug $\times$ INT interaction was not significant $\left(F_{(1,16)}=1.85\right.$; NS).

\section{Comparison of performance between smokers and nonsmokers}

In the absence of nicotine and with the amount of previous task exposure equated between groups (see Materials and Methods), performance of smokers never differed from that of nonsmokers. This was confirmed by the absence of a group main effect and of any interactions involving group in three-factor ANOVA with CUE and INT as additional factors, performed on RTval, RTinval, OMval, OMinval, and $\mathrm{RTval}_{\text {stdev }}$. In contrast, when comparing smokers with nicotine patch to controls with the same analysis, the effects of group interacted with INT on RTval, RTinval, and $\mathrm{RTval}_{\text {stdev }}\left(F_{(1,32)}>5.92\right.$; $p<0.05$ in all three cases). The profile of effects of group resembled the effects of drug within the smoking population [i.e., reductions in RTval, RTinval, and $\mathrm{RTval}_{\text {stdev }}$ were greater for $\mathrm{INT}_{\text {high }}$ (data not shown)]. No effects involving group were seen on OMval or OMinval.

\section{MRI}

Main effects of drug

Cue-only trials (representing cue-induced activity). Six clusters were identified that displayed a significant effect of drug across all cue conditions: one cluster in ACC and two in posterior cingulate cortex (PCC) [extending in one case into subparietal sulcus (SPS)], left angular gyrus, left anterior middle frontal gyrus (MFG), and

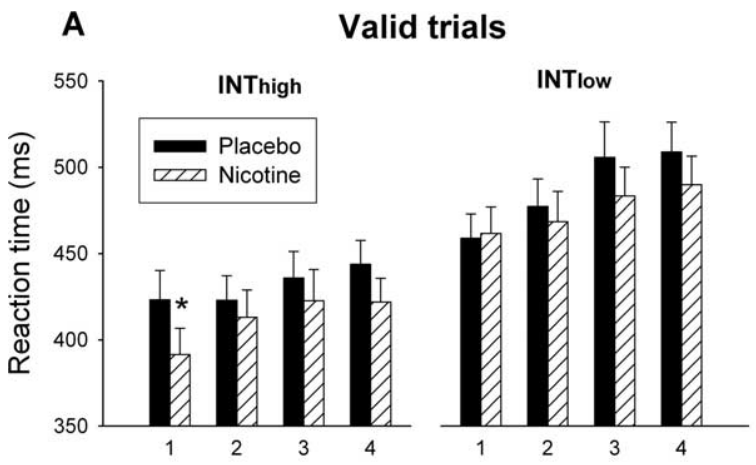

B Invalid trials
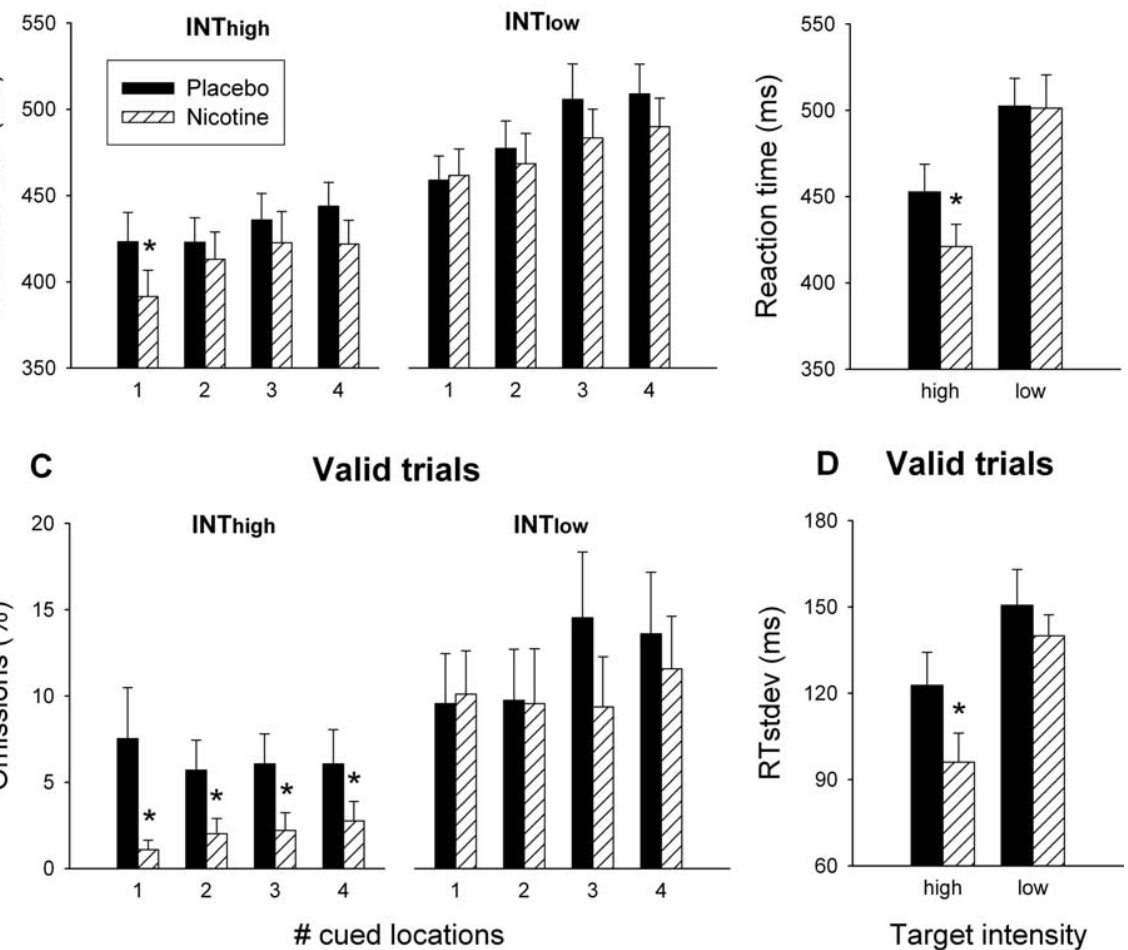

D Valid trials

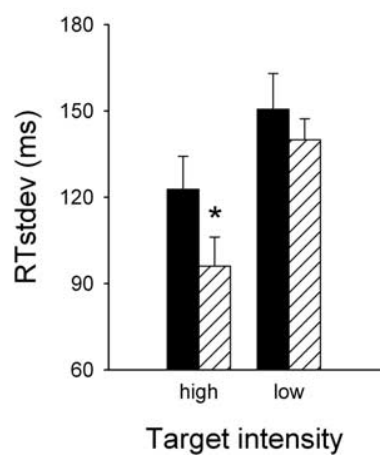

Figure 3. Effects of nicotine on performance of the SARAT. Nicotine (hatched bars) improved the RT of validly $(\boldsymbol{A})$ and invalidly cued trials $(\boldsymbol{B})$, omission errors $(\boldsymbol{C})$, and the SD of valid-trial $\mathrm{RT}(\boldsymbol{D})$ under specific task conditions. Data are presented as averages \pm SEM $(n=17)$. Conditions in which performance under nicotine differed significantly from that under placebo are marked $\left({ }^{*} p<\right.$ 0.05 ; paired $t$ test).

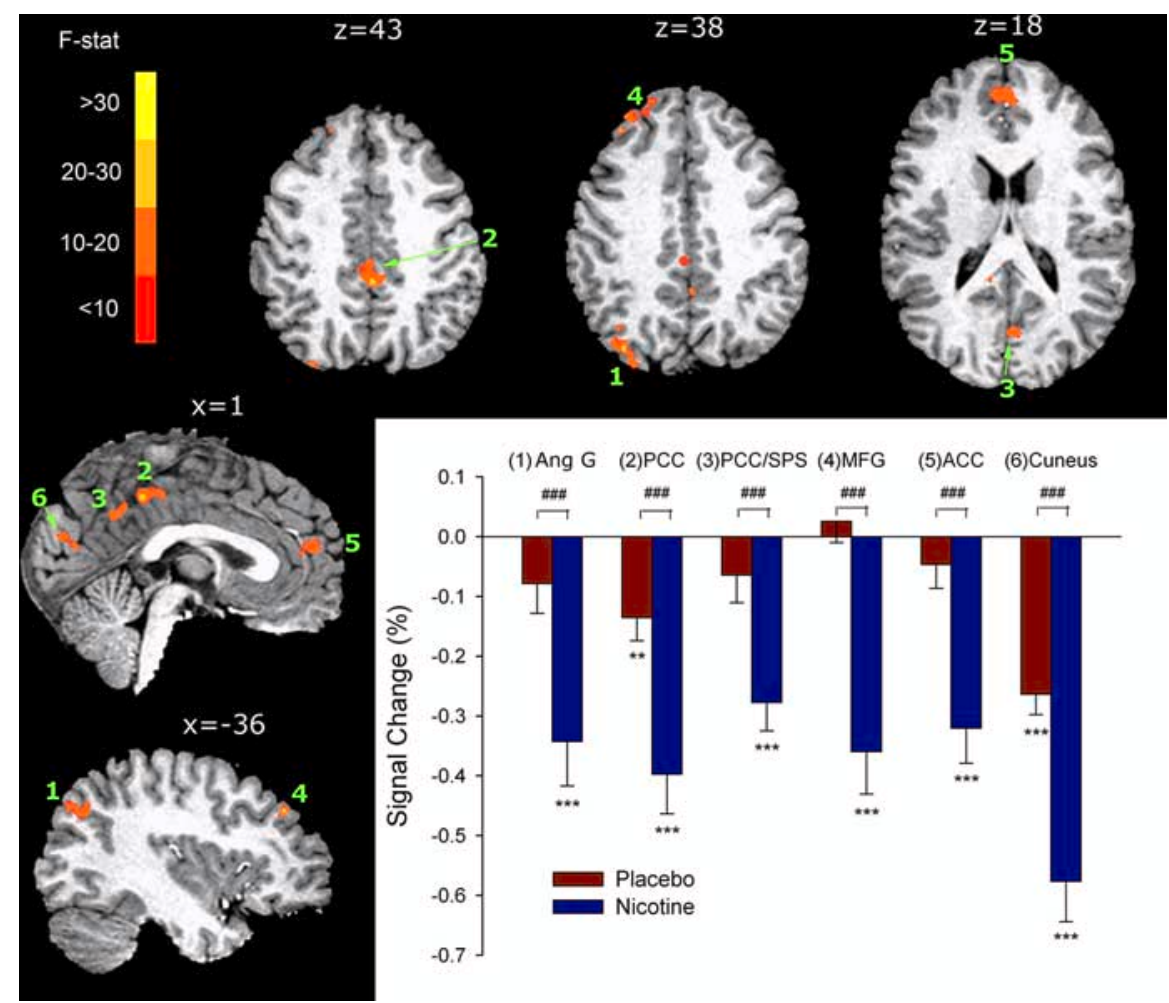

Figure 4. Main effects of nicotine in cue-only trials. Group activation maps are overlaid onto a rendered anatomical scan in Talairach space. In left angular gyrus (Ang G), PCC, SPS, left MFG, ACC, and cuneus, nicotine decreased the BOLD signal related to cue-only trials, causing significant deactivations. Data in the inset graph are presented as averages \pm SEM $(n=17)$. Significant differences from zero in one-sample $t$ tests $\left({ }^{* *} p<0.01 ;{ }^{* *} p<0.001\right)$ and significant differences between the nicotine and placebo sessions in paired $t$ tests $(\# \#) 00.001)$ are indicated. The numbering corresponds to ROIs in Table 2. F-stat, F statistic. 
Table 2. Brain regions identified by ANOVA of cue-only trials

\begin{tabular}{|c|c|c|c|c|c|}
\hline Brain region & & Side & Center of mass $(X, Y, Z$, in $\mathrm{mm})$ & Brodmann area(s) & Volume $(\mu \mathrm{l})$ \\
\hline \multicolumn{6}{|c|}{ Main effect of drug } \\
\hline 1 & Angular gyrus & $\mathrm{L}$ & $-33.3,-73.6,36.6$ & 39,7 & 733 \\
\hline 2 & Posterior cingulate gyrus and sulcus & $B$ & $0.4,-33.5,42.6$ & 23,31 & 687 \\
\hline 3 & Posterior cingulate gyrus and sulcus and subparietal sulcus & B & $-2.6,-47.8,30.1$ & $23,30,31$ & 633 \\
\hline 4 & Anterior middle frontal gyrus & L & $-26.0,44.6,34.5$ & $a^{20,001}$ & 598 \\
\hline 5 & Anterior cingulate gyrus and sulcus & $B$ & $-1.0,48.1,17.0$ & 32,24 & 477 \\
\hline 6 & Cuneus & B & $2.9,-74.5,20.3$ & 18 & 350 \\
\hline \multicolumn{6}{|c|}{ Interaction of drug $\times$ cue } \\
\hline & Anterior medial frontal gyrus & $\mathrm{R}$ & $3.4,51.3,27.9$ & 8,9 & 415 \\
\hline
\end{tabular}

Brain regions whose activity in cue-only trials of the SARAT was modulated by nicotine. The numbering corresponds to that of Figure 4. B, Bilateral; $L$, left; $R$, right.

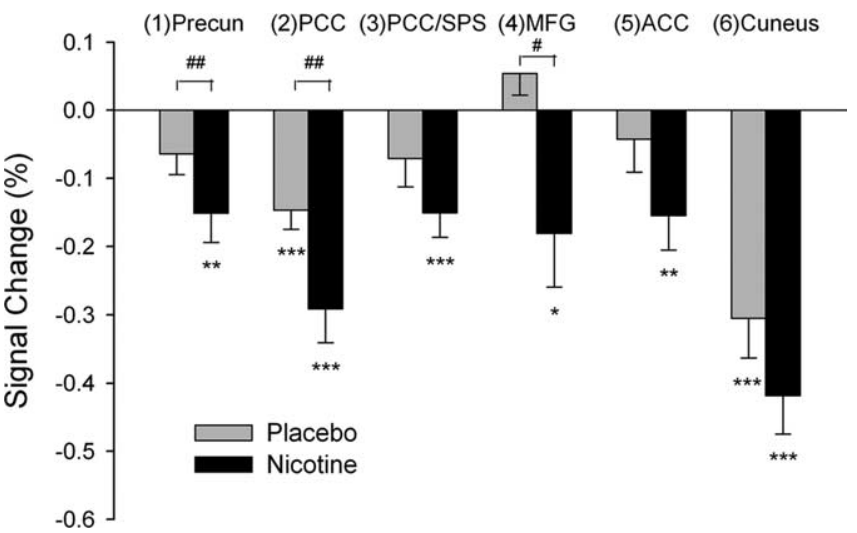

Figure 5. Effects of nicotine on activity in valid target trials within regions identified by analysis of cue-only trials. Activity in valid target trials was averaged within regions of the brain identified by voxelwise ANOVA of cue-only trials and compared between the nicotine and placebo sessions by paired $t$ tests $\left({ }^{\#} p<0.05\right.$; \#\# $\left.p<0.01\right)$. Significant differences from zero are indicated by asterisks ( ${ }^{*} p<0.05 ;{ }^{* *} p<0.01$; ${ }^{* * *} p<0.001$; one-sample $t$ test). Bars represent the average \pm SEM $(n=17)$. Precun, Precuneus.

bilateral cuneus (Fig. 4, Table 2). In all regions, nicotine either induced or enhanced cue-induced deactivations. In the absence of nicotine, the average BOLD signal never differed between smokers and nonsmokers ( ${ }_{1}^{1} t_{1(32)}^{1}<1.12$; NS for all regions). In contrast, BOLD signal differed between smokers with nicotine patch and nonsmokers $\left(t_{(32)}>2.47 ; p<0.05\right.$ for all regions), mirroring the within-subject effects of drug in smokers (data not shown).

Because cue-related processes also contribute to target trials, although terminated early by the appearance of the target, they should, to some extent, mirror the nicotine-induced deactivations in cue-only trials. Paired $t$ tests were performed on the average BOLD values from the same regions in valid target trials. Indeed, deactivations with nicotine were found in the angular gyrus, one of the PCC regions, and MFG $\left(t_{(16)}>2.91 ; p<0.05\right)$, with trends also in the other three clusters (Fig. 5). With $p$ values Bonferroni adjusted for six comparisons, only PCC showed significant deactivation, and effects in all other regions were reduced to trends.

Partial correlations controlling for nicotine plasma levels were calculated between the six regional BOLD effects and effects of nicotine on RTval, RTinval, and $\mathrm{RTval}_{\text {stdev }}$ under $\mathrm{INT}_{\text {high }}$ and $\mathrm{INT}_{\text {low }}$. For RTval for which drug effects differed between cue conditions, correlations were calculated separately for trials with 1CUE and 4CUE. Table 3 lists correlation coefficients for the PCC, PCC/SPS, and angular gyrus; no significant correlations were identified for the other clusters. Subjects who displayed greater deactivation by nicotine of cue-related signal in the PCC regions tended to show greater reduction in RTval, RTinval, and $\mathrm{RTval}_{\text {stdev }}$ under $\mathrm{INT}_{\text {high }}$ (Fig. 6). Deactivation of left angular gyrus was associated with a reduction in $\mathrm{RTval}_{\text {stdev }}$ selectively under $\mathrm{INT}_{\text {high }}$. Controlling for nicotine plasma levels had little overall effect, and correlations with and without these control variables never differed $(z<0.68 ; p>0.5)$.

We next analyzed the degree of overlap between the nicotineinduced deactivations and regions of the "default network" that typically deactivate with external task demands and are active at rest and in whose vicinity the above deactivations appear to be located. The first approach was based on data from a metaanalysis of nine positron emission tomography (PET) studies contrasting activation during visual information processing tasks and a passive control condition (Shulman et al., 1997), recently recomputed with updated methods (Buckner et al., 2005). A contrast image that averaged the active minus passive $\left[{ }^{15} \mathrm{O}\right] \mathrm{H}_{2} \mathrm{O}$ PET scans was overlaid onto the current activation map. Five of the six regions (the exception being cuneus) fully overlapped with areas that had been more active at rest than during task in the previous meta-analysis. When the PET data were thresholded at $>0.5 \%$ blood flow decrease, the ACC and PCC regions were $100 \%$ covered, the MFG region was $87 \%$ covered, and the angular gyrus was $39 \%$ covered. At $>1 \%$ decrease, the overlap covered $97-$ $100 \%$ of the ACC and PCC regions and 25 and $10 \%$ of the MFG and angular gyrus, respectively. The second approach was based on areas of deactivation induced by the current task, collapsed across study groups, sessions, and all active task trials. All six regions were located in areas that were less active with task $(100 \%$ overlap in ACC, PCC, and cuneus; $87 \%$ in MFG; and $96 \%$ in angular gyrus). At a voxelwise threshold of $p<0.01$ (one-sample $t$ test against zero; cluster volume, $\geq 665 \mu \mathrm{l}$ ), the overlap with task-negative regions was $100 \%$ for PCC and cuneus, $51 \%$ for PCC/SPS, $47 \%$ for ACC, $14 \%$ for MFG, and 26\% for angular gyrus.

Valid target trials. Three clusters were identified whose voxels displayed a significant main effect of drug in two-factor ANOVA with either CUE or INT as the second within-subject factor (Fig. 7, Table 4). A thalamic region was predominantly left-lateralized and covered medial association nuclei including mediodorsal nucleus; nicotine reduced activation in this region. It also deactivated a region spanning lingual gyrus and cerebellar declive, but because this region was mostly located within the interhemispheric cleft, this effect is not further analyzed or discussed. Finally, nicotine increased activation in the right MFG.

In the absence of nicotine, average BOLD signal in the thalamus did not differ between smokers and nonsmokers $\left(t_{(32)}=\right.$ $1.62 ; \mathrm{NS})$. In the MFG, activation was higher in nonsmokers than in smokers $\left(t_{(32)}=2.95 ; p<0.01\right)$, and nicotine reversed this difference (data not shown). However, interpreting these data as reversal of a nicotine deprivation-induced impairment is prob- 
Table 3. Correlations between the effects of nicotine on regional activity in cue-only trials and on performance indices

\begin{tabular}{|c|c|c|c|c|}
\hline & & (1) $L$ angular $G$ & (2) B PCC & (3) B PCC and SPS \\
\hline \multirow[t]{2}{*}{ RTval-1CUE } & $\mathrm{INT}_{\text {high }}$ & $r=0.58 ; \mathrm{NS}$ & $r=0.74 ; p<0.005$ & $r=0.89 ; p<0.001$ \\
\hline & INT $_{\text {low }}$ & $r=0.28 ; \mathrm{NS}$ & $r=0.51 ; \mathrm{NS}$ & $r=0.58 ; \mathrm{NS}$ \\
\hline \multirow[t]{2}{*}{ RTval-4CUE } & INT $_{\text {high }}$ & $r=0.56 ; \mathrm{NS}$ & $r=0.70 ; p<0.005$ & $r=0.45 ; \mathrm{NS}$ \\
\hline & $\mathrm{INT}_{\text {low }}$ & $r=0.23 ; \mathrm{NS}$ & $r=0.39 ; \mathrm{NS}$ & $r=0.61 ; \mathrm{NS}$ \\
\hline \multirow[t]{2}{*}{ RTinval } & $\mathrm{INT}_{\text {high }}$ & $r=0.47 ; \mathrm{NS}$ & $r=0.73 ; p<0.005$ & $r=0.68 ; p<0.005$ \\
\hline & INT $_{\text {low }}$ & $r=0.50 ; \mathrm{NS}$ & $r=0.40 ; \mathrm{NS}$ & $r=0.57 ; \mathrm{NS}$ \\
\hline RTval $_{\text {stdev }}$ & $\begin{array}{l}\text { INT }_{\text {high }} \\
\text { INT }_{\text {low }}\end{array}$ & $\begin{array}{l}r=0.76 ; p<0.001 \\
r=0.13 ; \mathrm{NS}\end{array}$ & $\begin{array}{l}r=0.73 ; p<0.005 \\
r=0.21 ; \mathrm{NS}\end{array}$ & $\begin{array}{l}r=0.67 ; p=0.006 ; \mathrm{NS} \\
r=0.40 ; \mathrm{NS}\end{array}$ \\
\hline
\end{tabular}

Angular G, Angular gyrus; L, left; B, bilateral. The numbering of regions corresponds to that in Figure 4 and Table 2.

lematic; a positive correlation between the effects of nicotine on BOLD signal and RTval under 1CUE-INT high $_{\text {indicates }}$ smaller RT benefits in subjects with larger nicotine-induced activation of this region. This correlation narrowly failed the $p<$ 0.005 significance level $(r=0.67 ; p=$ $0.007)$. None of the other BOLD effects of nicotine in valid target trials correlated with its performance effects in these trials.

Invalid target trials. Two posterior medial clusters displayed significant main effects of drug, one located in precuneus and one in cuneus bordering precuneus (Fig. 8 , Table 5). In both regions, the effects of nicotine consisted of reductions in activation to yield, in one case, a significant deactivation relative to the session baseline. However, neither of these deactivations correlated with performance effects of nicotine in invalid target trials. In the absence of nicotine, the average BOLD signal amplitude in these trials never differed between smokers and nonsmokers $\left({ }_{1}^{1} t_{1}^{1}{ }_{(32)}<\right.$ 1.70; NS for both regions).

Interactions of drug with task conditions

Cue-only trials. Only one cluster, located in right anterior medial frontal gyrus (Fig. 9, Table 2), was identified as displaying a drug $\times$ CUE interaction. In this region, nicotine induced significant deactivation only in the 2CUE and 3CUE trials.

Valid target trials. A cluster in bilateral cuneus (Fig. 10, Table 4) displayed a significant drug $\times$ CUE interaction, which was attributable to nicotine enhancing deactivation in 3CUE and 4CUE trials. Under placebo, deactivation progressively weakened with increasing CUE. No clusters were identified that displayed a significant drug $\times$ INT interaction.

Invalid target trials. Clusters displaying a significant drug $\times$ INT interaction were located in right MFG in the approximate vicinity but not overlapping with the MFG region that displayed a drug main effect in target trials, in left intraparietal sulcus (IPS) and in left lingual gyrus (Fig. 11, Table 5). In all three regions, nicotine decreased activation in trials with $\mathrm{INT}_{\text {high }}$ but increased it with $\mathrm{INT}_{\text {low }}$. Thus, although the BOLD signal never differed between $\mathrm{INT}_{\text {high }}$ and $\mathrm{INT}_{\text {low }}$ under placebo, it was always significantly larger under $\mathrm{INT}_{\text {low }}$ than $\mathrm{INT}_{\text {high }}$ in the presence of nicotine.

\section{Controls for nonspecific effects of nicotine on blood flow and coupling}

Within the visual and motor regions that responded to the flashing checkerboard and finger-tapping manipulation, neither
BOLD- nor flow-weighted contrast values (on vs off periods) differed between the nicotine and the placebo sessions $\left(t_{(13-16)}<\right.$ 1.61; NS for each region; paired $t$ tests). Thus, the presence of nicotine did not alter BOLD or CBF responses to neuronal stimulation in a nonspecific manner.

At rest, absolute quantitative blood flow averaged $71.7 \pm 15.4$ $\mathrm{ml} / 100 \mathrm{~g} / \mathrm{min}$ in gray and $38.6 \pm 11.1 \mathrm{ml} / 100 \mathrm{~g} / \mathrm{min}$ in white matter. These values did not differ between the nicotine and the placebo sessions $\left(t_{(16)}<1\right.$; paired $t$ tests; for both gray and white matter). Resting CBF also did not differ between the nicotine and placebo condition in any of the six ROIs (Table 2) identified in cue-only trials as being deactivated by nicotine $\left(t_{(13-16)}<1.25\right.$ for all regions except cuneus, in which $t_{(11)}=1.86$; NS for all regions).

\section{Discussion}

The main finding of this study is that nicotine induced or potentiated task-related deactivation in regions of the brain that overlapped with the so-called default network of resting brain function (Gusnard and Raichle, 2001) during performance of a visuospatial attention task. These deactivations were associated with performance improvements and cannot be explained by any nonspecific effects of nicotine on neurovascular coupling or basal CBF. 
Table 4. Brain regions identified by ANOVA of valid target trials

\begin{tabular}{|c|c|c|c|c|c|}
\hline Brain region & & Side & Center of mass $(X, Y, Z$, in $\mathrm{mm})$ & Brodmann area(s) & Volume $(\mu l)$ \\
\hline \multicolumn{6}{|c|}{ Main effect of drug } \\
\hline 1 & Thalamus & B & $-1.4,-22.4,10.6$ & & 1699 \\
\hline 2 & Lingual gyrus/declive (largely located in interhemispheric cleft) & B & $-2.9,-76.3,-9.6$ & 18 & 772 \\
\hline 3 & Middle frontal gyrus & $\mathrm{R}$ & $48.1,17.7,26.4$ & 9 & 391 \\
\hline \multicolumn{4}{|c|}{ Interaction drug $\times$ cue } & & 2365 \\
\hline
\end{tabular}

Regions of the brain whose activity in valid target trials was modulated by nicotine. The numbering corresponds to that of Figure 7. B, Bilateral; $R$, right.
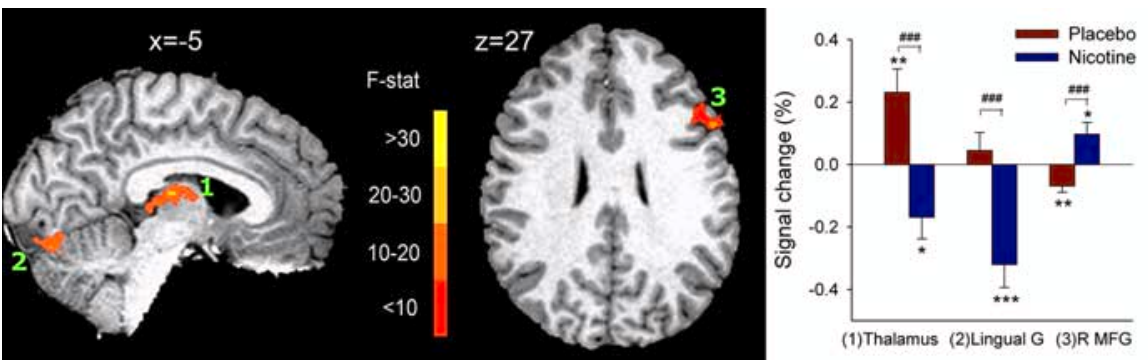

Figure 7. Main effects of nicotine in valid target trials. Nicotine deactivated the thalamus and lingual gyrus (G) but activated the right (R) MFG. Significant differences from zero in one-sample $t$ tests $\left({ }^{*} p<0.05 ;{ }^{* *} p<0.01 ;{ }^{* * *} p<0.001\right)$ and significant differences between the nicotine and placebo sessions in paired $t$ tests ( ${ }^{\# \#} p<0.001$ ) are indicated. The numbers correspond to Rols in Table 4. F-stat, F statistic.

A network of resting brain function was first suggested by a meta-analysis of PET studies (Shulman et al., 1997), in which a consistent set of brain regions displayed lower activity during visual processing tasks than in the absence of external task demands ("at rest"). Others have since confirmed similar networks that deactivate with attention-demanding tasks, most consistently the PCC and rostral ACC/medial prefrontal cortex (Binder et al., 1999; Mazoyer et al., 2001; McKiernan et al., 2003). Taskinduced deactivation appears to reflect suspension of cognitive processing that is ongoing in the control condition (Raichle et al., 2001), such as spontaneous semantic and self-referential processing, broad stimulus monitoring, and emotional processing (see also Gusnard et al., 2001; Simpson et al., 2001; Gilbert et al., 2006; Hahn et al., 2007).

Resting activity correlates between brain regions that routinely display task-induced deactivation, and these regions are anti-correlated to a task-positive network. These anti-correlated networks have been suggested to underlie competition between task-focused attention and stimulus-independent thought (Greicius et al., 2003; Fox et al., 2005). Polli et al. (2005) identified deactivation in default regions early during trials of an antisaccade task, consistent with a performance-optimizing function. Indeed, trials marked by a failure to acutely deactivate these regions were associated with errors, giving evidence of instant performance benefits of dynamically downregulating default functions. The present findings are in accordance with such a dynamic, trial-by-trial downregulation by nicotine. Deactivations were induced specifically in active task trials relative to baseline (i.e., they did not occur uniformly throughout runs). Also, the absence of nicotine effects on resting CBF, despite lower sensitivity of this measure, adds evidence against a tonic network downregulation. Thus, nicotine may facilitate neuronal mechanisms responsible for transitions from a default mode to one that favors responsiveness to external demands.

Nicotine deactivated task-related BOLD signal, or enhanced existing deactivations, specifically in cue-only trials, suggesting modulation of cue-induced processes. Such processes can be ex- pected to be most prominent in cue-only trials, in which they neither terminate with target onset nor interact with targetinduced processes. Cue-induced processes also contribute to target trials, and trends toward deactivation with nicotine were seen in the same regions in valid trials. Polli et al. (2005) emphasized the performance-optimizing function of these acute downregulations. Accordingly, robust associations between nicotineinduced deactivation of cue-related signal in default regions and performance enhancement were identified. Downregulation of default functions, being cueinduced, appears to precede the performance-enhancing effects of nicotine that were dependent on the nature of the target stimulus, i.e., selective for $\mathrm{INT}_{\text {high }}$ (cues precede targets).

One question is whether nicotine potentiated task-related deactivations directly or whether this effect was secondary to potentiation of task-related activations. In general, the data do not support a strengthening of task-related activity by nicotine, and in those instances in which activations were observed, they were not associated with performance improvements, as were the default deactivations. However, a possibility remains that some drug-induced increases in cuerelated activity, such as in topographically organized visual areas, were not detected by group analysis. Another possible secondary mode of action is that the default network was more active with nicotine at baseline, thus facilitating task-induced deactivation. The finding that resting CBF did not change with nicotine in any of the identified default regions speaks against that possibility, although the lower signal/noise of CBF measurements should be noted.

The effects of nicotine on default regions did not interact with CUE, suggesting that downregulation occurred as a general response to cues announcing targets and the need for preparatory cognitive processes. The general lack of preference of nicotine effects for any level of cue precision speaks against a modulation of visuospatial selective attention. On RTvalid, the drug effects interacted with INT only in 1CUE trials, but no interaction supported a preferential action in this cue condition. The lack of preference for trials in which the cue was informative speaks against a modulation of endogenous, cue-induced orienting. Furthermore, the lack of preference for trials in which the target was unpredictable and attentional shifts were triggered by its physical onset speaks against specific facilitation of stimulusdriven orienting.

The behavioral effects of nicotine displayed preference for $\mathrm{INT}_{\text {high }}$. It is noteworthy that a recent study reported reduced search times with nicotine specifically for signals of high physical salience (Rycroft et al., 2005); a study in rats yielded similar re- 
Table 5. Brain regions identified by ANOVA of invalid target trials

\begin{tabular}{|c|c|c|c|c|c|}
\hline Brain region & & Side & Center of mass $(X, Y, Z$, in $\mathrm{mm})$ & Brodmann area(s) & Volume $(\mu \mathrm{l})$ \\
\hline \multicolumn{6}{|c|}{ Main effect of drug } \\
\hline 1 & Medial precuneus & B & $2.2,-46.9,52.4$ & 7 & 482 \\
\hline 2 & Cuneus, precuneus & $\mathrm{R}$ & $8.0,-76.7,39.0$ & 19,7 & 383 \\
\hline \multicolumn{6}{|c|}{ Interaction of drug $\times$ target } \\
\hline 1 & Middle frontal gyrus and inferior frontal sulcus & $\mathrm{R}$ & $38.2,13.3,35.5$ & 8,9 & 733 \\
\hline 2 & Intraparietal sulcus and inferior parietal lobule & L & $-31.6,-46.2,37.6$ & 40 & 436 \\
\hline 3 & Lingual gyrus & $\mathrm{L}$ & $-23.5,-69.4,-9.9$ & $18 / 19$ & 328 \\
\hline
\end{tabular}

Regions of the brain whose activity in invalid target trials was modulated by nicotine. The numbering corresponds to that of Figures 8 and 11. B, Bilateral; L, left; R, right.
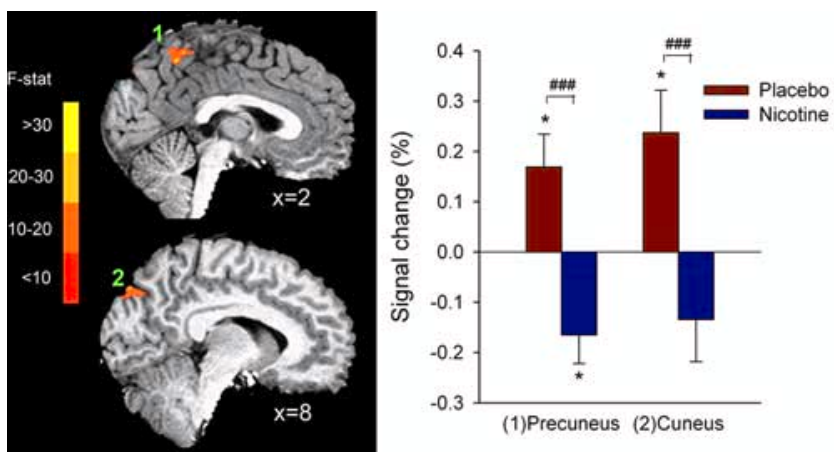

Figure 8. Main effects of nicotine in invalid target trials. Nicotine deactivated the cuneus and precuneus. Significant differences from zero $\left({ }^{*} p<0.05\right.$; one-sample $t$ test $)$ and between nicotine and placebo ( ${ }^{\# \#} p<0.001$; paired $t$ test) are indicated. The numbers correspond to ROIs in Table 5. F-stat, F statistic.

sults (Hahn et al., 2002). These findings seem to exclude a mechanism of action via enhanced visual processing sensitivity. Instead, nicotine appears to facilitate orienting to physically salient events. One major difference between stimuli with varying physical intensities lies in their alerting properties. Low-intensity targets in the present study were faint, and although they were detected in $85-90 \%$ of trials, they can be expected to be much less alerting than high-intensity targets. Nicotine may thus potentiate the alerting function exerted by external stimuli; such potentiation would be expected to be more pronounced with stimuli that are already alerting. This explanation would resonate with a shift from task-independent thought to externally oriented information processing. Enhanced downregulation of default functions in response to cues may have increased the alerting properties of target stimuli.

This interpretation is also compatible with the literature on the type of attention most affected by nicotine. Improvements have been reported primarily in nonelaborate stimulus detection (see Introduction), in line with an enhanced orientation of information-processing resources toward external events. Evidence that nicotine helps sustain attention over prolonged periods of time is consistent with a deactivation of default functions; when attention toward a task starts to dwindle and taskindependent thought processes intrude, nicotine may help impede such internal processes and maintain the alerting properties of the task stimuli. An enhanced alerting function of external signals is also in accordance with a facilitation of attentional shifts toward signals at previously unattended locations (see Introduction).

Decreased CBF in ACC has previously been reported after nicotine administration (Ghatan et al., 1998; Ernst et al., 2001). However, the present results differ from reports of enhanced activity in regions associated with visual attention and arousal (Lawrence et al., 2002; Thiel et al., 2005). The paucity of regional
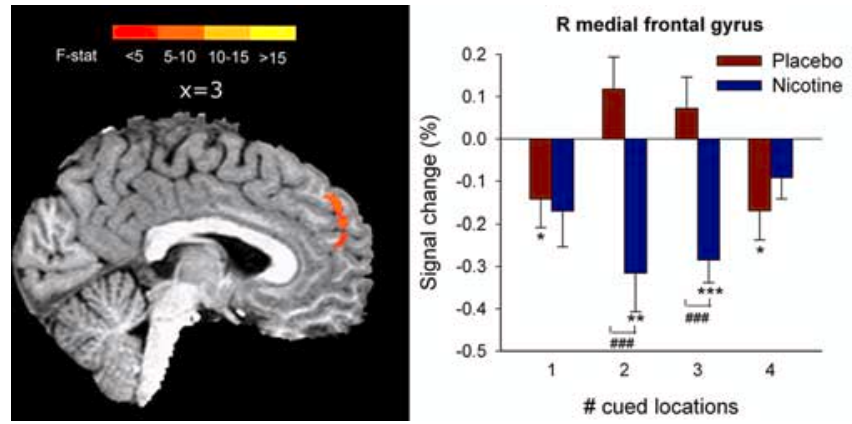

Figure 9. Interaction of the effects of nicotine with the number of cued locations in cue-only trials. Nicotine induced deactivations in right $(\mathrm{R})$ medial frontal gyrus only in trials with two and three cued locations. Significant differences from zero $\left({ }^{*} p<0.05 ;{ }^{* *} p<0.01\right.$; ${ }^{* * *} p<0.001$; one-sample $t$ test) and between nicotine and placebo $\left({ }^{\# \# \#} p<0.001\right.$; paired $t$ test) are indicated. F-stat, Fstatistic.
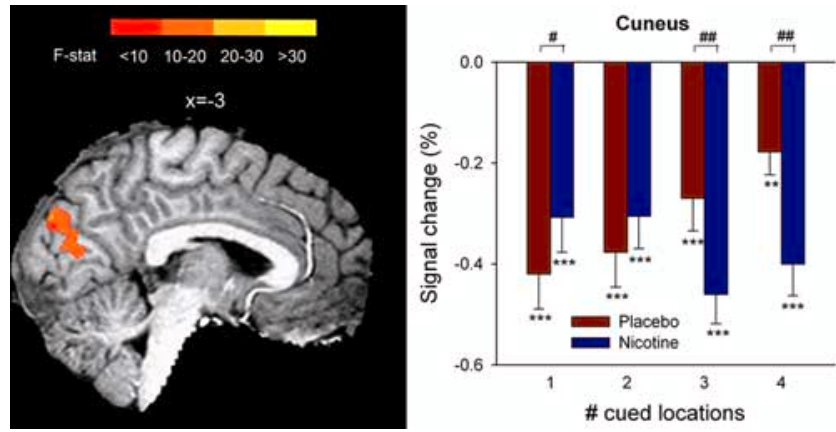

Figure 10. Interaction of the effects of nicotine with the number of cued locations in valid target trials. In medial cuneus, nicotine potentiated deactivation in valid trials with three and four cued locations. Significant differences from zero $\left({ }^{* *} p<0.01\right.$; ${ }^{* *} p<0.001$; one-sample $t$ test) and between nicotine and placebo $\left({ }^{\#} p<0.05\right.$; ${ }^{\#} p<0.01$; paired $t$ test) are indicated. F-stat, Fstatistic.

activations with nicotine was surprising, although their location (MFG, IPS, and lingual gyrus) was consistent with areas involved in visual attention modulated by nicotine (see above). Most activations were seen in invalid trials with $\mathrm{INT}_{\text {low }}$, the most difficult task condition. Parietal and thalamic activation by nicotine during the RVIP task, its absence in a low-level control task (Lawrence et al., 2002), and its general absence and even deactivation in the SARAT, may thus reflect differences in task difficulty. Although the SARAT engages areas of the frontoparietal attention network (Hahn et al., 2006), the level of activity within these regions may not be the main performance-limiting factor. Indeed, enhanced MFG activation by nicotine tended to be associated with slower RTval in a condition in which nicotine overall reduced RT. Similarly, RT reductions were seen in invalid trials with $\mathrm{INT}_{\text {high }}$, in which nicotine reduced activation in MFG, IPS, 


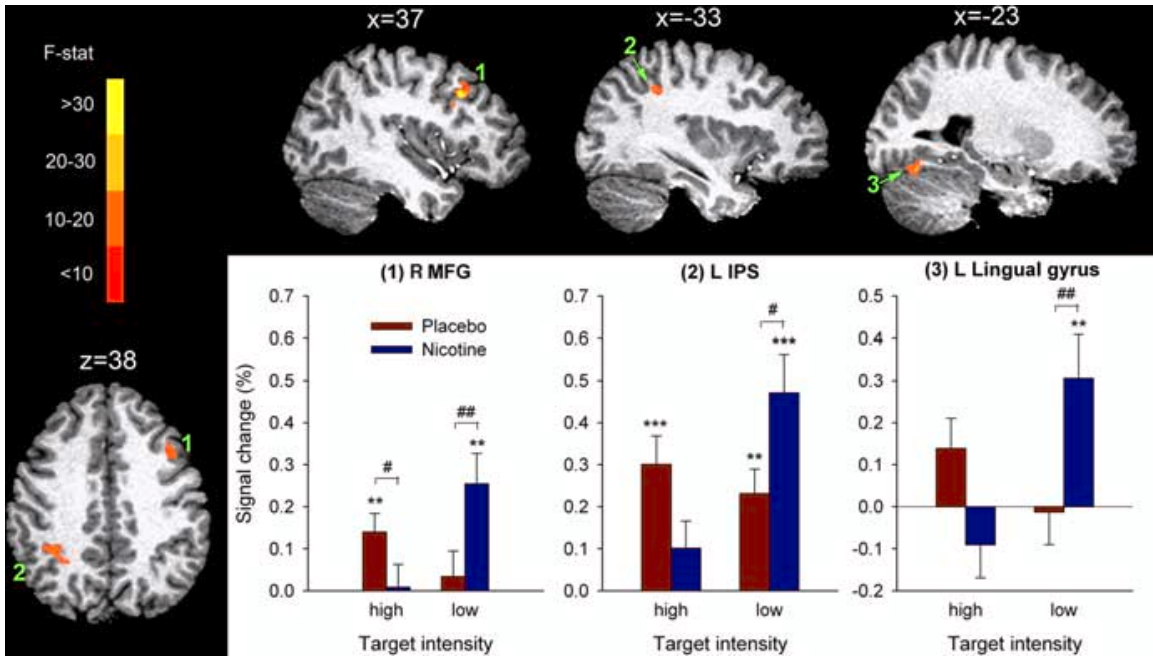

Figure 11. Interaction of the effects of nicotine with target intensity in invalid target trials. Nicotine increased activity in the right (R) MFG, left IPS, and left (L) lingual gyrus in invalid trials with low target intensity but tended to decrease activity in trials with high target intensity (effect significant in MFG). Significant differences from zero ${ }^{* *} p<0.01$; ${ }^{* * *} p<0.001$; one-sample $t$ test) and between nicotine and placebo ( ${ }^{\#} p<0.05$; ${ }^{\# \#} p<0.01$; paired $t$ test) are indicated. F-stat, F statistic. smokers may only be determined after periods of application long enough to develop tolerance to its adverse effects (Heishman et al., 1993; Perkins et al., 1994). One immediate implication of the present findings is that potentiation of task-induced default deactivation is likely to contribute toward the maintenance of nicotine self-administration, probably helping smokers to stay "on task." Although the blind application of nicotine and placebo patches could increase introspection and other processes related to default functions, the deactivations were not specific to a situation of elevated default activity, because they were seen also when comparing smokers with nicotine patch to nonsmokers without patch.

Nicotine has well known sympathomimetic properties (Perkins et al., 2004; Yugar-Toledo et al., 2005) (see also HR increase in the present study) and can and lingual gyrus, but not with $\mathrm{INT}_{\text {low, }}$ in which it increased activity in these regions.

This raises the possibility that other deactivations by nicotine (thalamus in valid trials and cuneus and precuneus in invalid trials) were adaptive in the behavioral context set by the task, perhaps reflecting reduced effort or increased automaticity underlying performance. Deactivation in cuneus (Fig. 10) could reflect decreased visual representation of the cue location when attentional resources were shifted to the target locations, and indeed, deactivation increased with cue precision. Nicotine potentiated this deactivation in 3CUE and 4CUE trials, in which attentional orienting away from the center likely was driven mostly by target onset. In cue-only trials, nicotine induced deactivations in the medial frontal gyrus in 2CUE and 3CUE trials, conditions in which cues are informative and evoke attentional orienting, but attention is split between several target locations. This may have created a conflict situation, and nicotine may have deemphasized such processes.

The finding that performance and regional activation generally did not differ between nonsmokers and smokers in the absence of nicotine suggests that the nicotine effects did not constitute a restoration of normal function in mildly deprived smokers. Smokers' subjective self-reports did not indicate any nicotinewithdrawal symptoms. Smokers reported higher craving levels after than before scans; the presence or absence of nicotine replacement had no effect thereon. The fact that all participants reported to be more tired and less contented and focused after than before scans suggests that general fatigue and dysphoric effects of the 2-h test sessions were responsible. Smokers rated themselves as more focused in the nicotine session, consistent with the observed performance enhancement. This did not reflect detrimental effects of nicotine deprivation, because measurements in nonsmokers and smokers in the absence of nicotine never differed.

The above does not imply that nonsmokers would necessarily display an identical profile of effects. Although there is no evidence for qualitative differences between the effects of nicotine in smokers and nonsmokers, additional studies will need to establish whether the findings generalize to drug-naive individuals and to clinical populations. The clinical value of nicotine in non- exert direct vascular effects (Toda, 1975; Boyajian and Otis, 2000; Sabha et al., 2000) that may alter BOLD responses (Bruhn et al., 1994, 2001; Wang et al., 2006). However, neither BOLD nor CBF responses to visuomotor stimulation were modulated by nicotine, suggesting the absence of nonspecific hemodynamic alterations, although a possibility remains that such effects are topographical in nature. Furthermore, absolute resting $\mathrm{CBF}$ did not differ between the nicotine and placebo sessions within gray or white matter or in any of the default regions deactivated by nicotine, consistent with results obtained using ${ }^{133}$ Xenon inhalation (Ghatan et al., 1998). Together, the observed effects of nicotine on task-related BOLD responses do not appear to be caused by direct effects on blood flow or neurovascular coupling (see also Jacobsen et al., 2002).

In summary, the present findings suggest that nicotine improves attentional performance by aiding the downregulation of so-called default functions of the brain in response to task-related cues. Future studies should investigate the possibility that other psychostimulant drugs may exert their performance-enhancing effects by a similar mechanism.

\section{References}

Bates T, Mangan G, Stough C, Corballis P (1995) Smoking, processing speed and attention in a choice reaction time task. Psychopharmacology (Berl) 120:209-212.

Bentley P, Husain M, Dolan RJ (2004) Effects of cholinergic enhancement on visual stimulation, spatial attention, and spatial working memory. Neuron 41:969-982.

Binder JR, Frost JA, Hammeke TA, Bellgowan PS, Rao SM, Cox RW (1999) Conceptual processing during the conscious resting state. A functional MRI study. J Cogn Neurosci 11:80-95.

Boyajian RA, Otis SM (2000) Acute effects of smoking on human cerebral blood flow: a transcranial Doppler ultrasonography study. J Neuroimaging 10:204-208.

Bruhn H, Kleinschmidt A, Boecker H, Merboldt KD, Hanicke W, Frahm J (1994) The effect of acetazolamide on regional cerebral blood oxygenation at rest and under stimulation as assessed by MRI. J Cereb Blood Flow Metab 14:742-748.

Bruhn H, Fransson P, Frahm J (2001) Modulation of cerebral blood oxygenation by indomethacin: MRI at rest and functional brain activation. J Magn Reson Imaging 13:325-334.

Buckner RL, Snyder AZ, Shannon BJ, LaRossa G, Sachs R, Fotenos AF, Sheline 
YI, Klunk WE, Mathis CA, Morris JC, Mintun MA (2005) Molecular, structural, and functional characterization of Alzheimer's disease: evidence for a relationship between default activity, amyloid, and memory. J Neurosci 25:7709-7717.

Bushnell PJ, Oshiro WM, Padnos BK (1997) Detection of visual signals by rats: effects of chlordiazepoxide and cholinergic and adrenergic drugs on sustained attention. Psychopharmacology (Berl) 134:230-241.

Corbetta M, Shulman GL (2002) Control of goal-directed and stimulusdriven attention in the brain. Nat Rev Neurosci 3:201-215.

Cox RW (1996) AFNI: software for analysis and visualization of functional magnetic resonance neuroimages. Comput Biomed Res 29:162-173.

Desimone R, Duncan J (1995) Neural mechanisms of selective visual attention. Annu Rev Neurosci 18:193-222.

Ernst M, Matochik JA, Heishman SJ, Van Horn JD, Jons PH, Henningfield JE, London ED (2001) Effect of nicotine on brain activation during performance of a working memory task. Proc Natl Acad Sci USA 98:4728-4733.

Foulds J, Stapleton J, Swettenham J, Bell N, McSorley K, Russell MA (1996) Cognitive performance effects of subcutaneous nicotine in smokers and never-smokers. Psychopharmacology (Berl) 127:31-38.

Fox MD, Snyder AZ, Vincent JL, Corbetta M, Van Essen DC, Raichle ME (2005) The human brain is intrinsically organized into dynamic, anticorrelated functional networks. Proc Natl Acad Sci USA 102:9673-9678.

Ghatan PH, Ingvar M, Eriksson L, Stone-Elander S, Serrander M, Ekberg K, Wahren J (1998) Cerebral effects of nicotine during cognition in smokers and non-smokers. Psychopharmacology (Berl) 136:179-189.

Giessing C, Thiel CM, Rosler F, Fink GR (2006) The modulatory effects of nicotine on parietal cortex activity in a cued target detection task depend on cue reliability. Neuroscience 137:853-864.

Gilbert SJ, Simons JS, Frith CD, Burgess PW (2006) Performance-related activity in medial rostral prefrontal cortex (area 10) during low-demand tasks. J Exp Psychol Hum Percept Perform 32:45-58.

Greicius MD, Krasnow B, Reiss AL, Menon V (2003) Functional connectivity in the resting brain: a network analysis of the default mode hypothesis. Proc Natl Acad Sci USA 100:253-258.

Grilly DM, Simon BB, Levin ED (2000) Nicotine enhances stimulus detection performance of middle- and old-aged rats: a longitudinal study. Pharmacol Biochem Behav 65:665-670.

Gusnard DA, Raichle ME (2001) Searching for a baseline: functional imaging and the resting human brain. Nat Rev Neurosci 2:685-694.

Gusnard DA, Akbudak E, Shulman GL, Raichle ME (2001) Medial prefrontal cortex and self-referential mental activity: relation to a default mode of brain function. Proc Natl Acad Sci USA 98:4259-4264.

Hahn B, Shoaib M, Stolerman IP (2002) Nicotine-induced enhancement of attention in the five-choice serial reaction time task: the influence of task demands. Psychopharmacology (Berl) 162:129-137.

Hahn B, Sharples CG, Wonnacott S, Shoaib M, Stolerman IP (2003) Attentional effects of nicotinic agonists in rats. Neuropharmacology 44:1054-1067.

Hahn B, Ross TJ, Stein EA (2006) Neuroanatomical dissociation between bottom-up and top-down processes of visuospatial selective attention. NeuroImage 32:842-853.

Hahn B, Ross TJ, Stein EA (2007) Cingulate activation increases dynamically with response speed under stimulus unpredictability. Cereb Cortex, in press.

Heishman SJ, Henningfield JE (2000) Tolerance to repeated nicotine administration on performance, subjective, and physiological responses in nonsmokers. Psychopharmacology (Berl) 152:321-333.

Heishman SJ, Snyder FR, Henningfield JE (1993) Performance, subjective, and physiological effects of nicotine in non-smokers. Drug Alcohol Depend 34:11-18.

Heishman SJ, Singleton EG, Moolchan ET (2003) Tobacco Craving Questionnaire: reliability and validity of a new multifactorial instrument. Nicotine Tob Res 5:645-654.

Jacobsen LK, Gore JC, Skudlarski P, Lacadie CM, Jatlow P, Krystal JH (2002) Impact of intravenous nicotine on BOLD signal response to photic stimulation. Magn Reson Imaging 20:141-145.

Kim I, Huestis MA (2006) A validated method for the determination of nicotine, cotinine, trans-3' -hydroxycotinine, and norcotinine in human plasma using solid-phase extraction and liquid chromatography- atmospheric pressure chemical ionization-mass spectrometry. J Mass Spectrom 41:815-821.

Kim SG (1995) Quantification of relative cerebral blood flow change by flow-sensitive alternating inversion recovery (FAIR) technique: application to functional mapping. Magn Reson Med 34:293-301.

Koelega HS (1993) Stimulant drugs and vigilance performance: a review. Psychopharmacology (Berl) 111:1-16.

Lawrence NS, Ross TJ, Stein EA (2002) Cognitive mechanisms of nicotine on visual attention. Neuron 36:539-548.

Levin ED, Rezvani AH (2002) Nicotinic treatment for cognitive dysfunction. Curr Drug Targets CNS Neurol Disord 1:423-431.

Mancuso G, Lejeune M, Ansseau M (2001) Cigarette smoking and attention: processing speed or specific effects? Psychopharmacology (Berl) 155:372-378.

Mazoyer B, Zago L, Mellet E, Bricogne S, Etard O, Houde O, Crivello F, Joliot M, Petit L, Tzourio-Mazoyer N (2001) Cortical networks for working memory and executive functions sustain the conscious resting state in man. Brain Res Bull 54:287-298.

McKiernan KA, Kaufman JN, Kucera-Thompson J, Binder JR (2003) A parametric manipulation of factors affecting task-induced deactivation in functional neuroimaging. J Cogn Neurosci 15:394-408.

Murphy FC, Klein RM (1998) The effects of nicotine on spatial and nonspatial expectancies in a covert orienting task. Neuropsychologia 36:1103-1114.

Newhouse PA, Potter A, Singh A (2004) Effects of nicotinic stimulation on cognitive performance. Curr Opin Pharmacol 4:36-46.

Parrott AC, Craig D (1992) Cigarette smoking and nicotine gum (0, 2 and 4 $\mathrm{mg}$ ): effects upon four visual attention tasks. Neuropsychobiology 25:34-43.

Parrott AC, Garnham NJ, Wesnes K, Pincock C (1996) Cigarette smoking and abstinence: comparative effects upon cognitive task performance and mood state over 24 hours. Hum Psychopharmacol 11:391-400.

Perkins KA, Grobe JE, Fonte C, Goettler J, Caggiula AR, Reynolds WA, Stiller RL, Scierka A, Jacob RG (1994) Chronic and acute tolerance to subjective, behavioral and cardiovascular effects of nicotine in humans. J Pharmacol Exp Ther 270:628-638.

Perkins KA, Lerman C, Keenan J, Fonte C, Coddington S (2004) Rate of nicotine onset from nicotine replacement therapy and acute responses in smokers. Nicotine Tob Res 6:501-507.

Phillips JM, McAlonan K, Robb WG, Brown VJ (2000) Cholinergic neurotransmission influences covert orientation of visuospatial attention in the rat. Psychopharmacology (Berl) 150:112-116.

Polli FE, Barton JJ, Cain MS, Thakkar KN, Rauch SL, Manoach DS (2005) Rostral and dorsal anterior cingulate cortex make dissociable contributions during antisaccade error commission. Proc Natl Acad Sci USA 102:15700-15705.

Poltavski DV, Petros T (2006) Effects of transdermal nicotine on attention in adult non-smokers with and without attentional deficits. Physiol Behav $87: 614-624$.

Raichle ME, MacLeod AM, Snyder AZ, Powers WJ, Gusnard DA, Shulman GL (2001) A default mode of brain function. Proc Natl Acad Sci USA 98:676-682.

Rycroft N, Rusted JM, Hutton SB (2005) Acute effects of nicotine on visual search tasks in young adult smokers. Psychopharmacology (Berl) 181:160-169.

Sabha M, Tanus-Santos JE, Toledo JC, Cittadino M, Rocha JC, Moreno Jr H (2000) Transdermal nicotine mimics the smoking-induced endothelial dysfunction. Clin Pharmacol Ther 68:167-174.

Shulman GL, Fiez JA, Corbetta M, Buckner RL, Miezin FM, Raichle ME, Petersen SE (1997) Common blood flow changes across visual tasks: II. Decreases in cerebral cortex. J Cogn Neurosci 9:648-663.

Simpson Jr JR, Drevets WC, Snyder AZ, Gusnard DA, Raichle ME (2001) Emotion-induced changes in human medial prefrontal cortex: II. During anticipatory anxiety. Proc Natl Acad Sci USA 98:688-693.

Stolerman IP, Mirza NR, Shoaib M (1995) Nicotine psychopharmacology: addiction, cognition and neuroadaptation. Med Res Rev 15:47-72.

Talairach J, Tournoux P (1988) Co-planar stereotaxic atlas of the human brain. New York: Thieme.

Thiel CM, Zilles K, Fink GR (2005) Nicotine modulates reorienting of visuospatial attention and neural activity in human parietal cortex. Neuropsychopharmacology 30:810-820. 
Toda N (1975) Nicotine-induced relaxation in isolated canine cerebral arteries. J Pharmacol Exp Ther 193:376-384.

Turchi J, Holley LA, Sarter M (1995) Effects of nicotinic acetylcholine receptor ligands on behavioral vigilance in rats. Psychopharmacology (Berl) 118:195-205.

Wang R, Foniok T, Wamsteeker JI, Qiao M, Tomanek B, Vivanco RA, Tuor UI (2006) Transient blood pressure changes affect the functional magnetic resonance imaging detection of cerebral activation. NeuroImage 31:1-11.

Wechsler D (1999) Wechsler abbreviated scale of intelligence, Ed 3. San Antonio, TX: The Psychological Corporation.

Witte EA, Davidson MC, Marrocco RT (1997) Effects of altering brain cholinergic activity on covert orienting of attention: comparison of monkey and human performance. Psychopharmacology (Berl) 132:324-334.

Wong EC, Buxton RB, Frank LR (1998) Quantitative imaging of perfusion using a single subtraction (QUIPSS and QUIPSS II). Magn Reson Med 39:702-708.

Yang S, Ross TJ, Zhang Y, Stein EA, Yang Y (2005) Head motion suppression using real-time feedback of motion information and its effects on task performance in fMRI. NeuroImage 27:153-162.

Yugar-Toledo JC, Ferreira-Melo SE, Sabha M, Nogueira EA, Coelho OR, Consolin Colombo FM, Irigoyen MC, Moreno Jr H (2005) Blood pressure circadian rhythm and endothelial function in heavy smokers: acute effects of transdermal nicotine. J Clin Hypertens (Greenwich) 7:721-728. 